\title{
Langerhans Cell Histiocytosis - A Case Report
}

Gurubacharya SMi, Gurubacharya RL ${ }^{2}$

${ }^{1}$ Dr. Simmi M Gurubacharya, MD Paediatrics, Lecturer, ${ }^{2}$ Dr. Rajesh L Gurubacharya, MD Paediatrics, Assistant Professor, Department of Pediatrics, College of Medical Sciences, Kathmandu University.

Address for Correspondence: Dr. Simmi Misra Gurubacharya,

E-mail:sm_pul@rediffmail.com

\begin{abstract}
Histiocytosis is a heterogenous group of disorders that are characterized by proliferation and activation of mononuclear phagocyte system. Langerhans Cell Histiocytosis (LCH) or Class I histiocytosis is a rare disorder of unknown etiology with proliferation of Langerhan cells which may infiltrate a single or multiple organs. This disease is more common in infants and children. It is usually sporadic but a familial pattern is known. The term embraces the whole clinical spectrum of the disorder from single bone lesions (eosinophilic granuloma) to an aggressive widespread multisystem disease in very sick child (Letterer-Siwe disease) with a wide variety of intermediate forms including the Hand-shuller Christian triad. The cause of LCH is not firmly established and most investigators in the field have long suspected that LCH is immunologic disorder either in its etiology or in its pathophysiology ${ }^{1,2}$. Recent evidence suggests that $\mathrm{LCH}$ is a clonal disorder rather than reactive disease ${ }^{3}$. $\mathrm{LCH}^{\mathrm{C}}$ is classified according to sites of involvement into single system disease and multisystem disease ${ }^{4}$. Single system disease can be either unifocal or multifocal. Multisystem disease can be either without organ dysfunction or with organ dysfunction. Clinical course of $\mathrm{LCH}$ with single system disease is usually benign with high chance of survival ${ }^{5,6}$. However, its clinical course is often unpredictable and patients can experience spontaneous remission and exacerbations. Histiocytic diseases are currently classified by the writing group of the Histiocyte Society ${ }^{7}$ in the three classes, namely;
\end{abstract}

Class I: Langerhans Cell Histiocytosis (LCH)

Class II: Histiocytosis of mononuclear phagocytes other than Langerhans Cells

Class III: Malignant Histiocytic disorders

Key words: Langerhans Cell Histiocytosis, Eosinophilic granuloma, Skull, lytic lesion

\section{Case Report}

A 2-year-old female child, hailing from Chitwan, was admitted to College of Medical Sciences, Bharatpur, Nepal with complaints of fever off and on for 6 months, left knee joint pain for 30 days, difficulty in walking with history of excessive perspiration for 20 days. There was no history of trauma, fall, features suggestive of bleeding and coagulation disorders, rashes over the body, cough, tachypnoea, cyanosis, jaundice, any urinary problems, neurological deficit, loss of consciousness, convulsions, hematemesis, melaena, drug intake, blood transfusion, tubercular contact, any septic foci over the body like ear discharge, scalp infection. According to the mother, child was born at home; birth weight was not known but was of average size. His immunization was complete according to EPI schedule. All developmental milestones occurred at appropriate age. Child was of average built. His height, weight and head circumference were within normal limits.

On examination; his pulse was $130 / \mathrm{min}$, respiratory rate 26/min, temperature $1010 \mathrm{~F}$ (Left Axilla) and BP100/60 $\mathrm{mmHg}$. He was severely anaemic and anicteric. Lymph nodes were not palpable. Rest of general examination was normal. Systemic examinations did not reveal any abnormalities. However, child was investigated on the line of PUO with severe anaemia. His haemoglobin, total count, differential count and ESR were $4.8 \mathrm{gm} / \mathrm{dl}, 14,100 / \mathrm{mm} 3$, N89, L10, E01 and $66 \mathrm{~mm}$ in the first hour respectively. Peripheral blood film did not show any abnormalities. Platelet count was $270,000 / \mathrm{mm} 3$. Reticulocyte count was $0.4 \%$. Bleeding time, Prothrombin time, APTT, liver and kidney function tests, urine routine, stool routine were within normal limits. Rheumatoid factor, ANA, HIV, Mantoux test, widal, blood culture and sensitivity were also negative. USG abdomen including hip and knee, chest X-ray, X-ray left knee and hip bilaterally showed no abnormalities. However, CRP was positive. Bone marrow aspirate done revealed hypoplasia of erythroid cells only. No other abnormalities seen. There was no evidence of leukemia. AFB smear and culture were also negative. Child was given blood transfusion for severe anemia, NSAIDS (Ibuprofen, Diclofenac) for joint pain and supportive treatment. However, child showed slight improvement in terms of relief of symptoms only. Parents wanted to take the child to Kathmandu, Nepal for further evaluation and given discharge on request. But there also, no diagnosis could be made with all available investigations and facilities.

After 1 month, child was brought again with similar complaints as mentioned above along with abdominal distension more in left hypochondriac region and pain over lower limbs bilaterally which was worse than before as additional fresh problems. On examination, child was severely anaemic with palpable cervical, axillary and inguinal lymph nodes, which were significant in size. Rest of general examination was normal. On abdominal examination, spleen 
was palpable $3 \mathrm{~cm}$ below left subcostal margin along spleenic axis, which was firm, smooth and non-tender. Liver was 1.5 $\mathrm{cm}$ palpable below right subcostal margin, which was firm, smooth and non-tender. Other systemic examinations did not reveal any abnormalities. The haemoglobin was $6 \mathrm{gm} / \mathrm{dl}$ and other parameters investigated as before were all normal. Abdominal sonography showed hepatosplenomegaly. Chest X-ray and X-ray hip bilaterally showed multiple lytic lesions over the upper end of left humerus, iliac bones, upper end and shaft of both femurs with expansion of medullary cavity (Figure1). The skull radiograph showed classical multiple punched out lesions (Figure2).

Multiple Lytic Lesions as Seen in The X-Rays

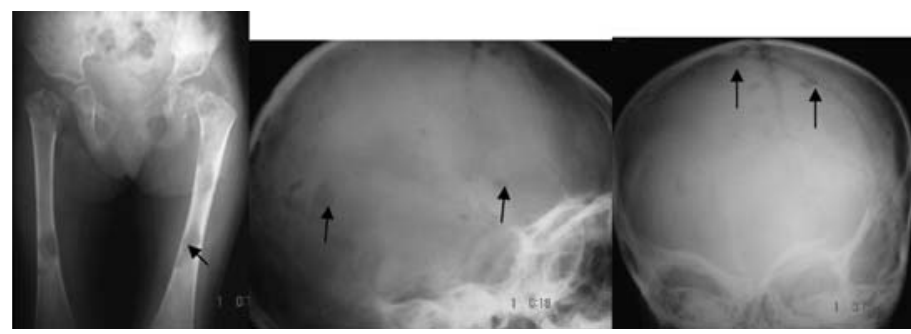

Fig 1

Fig 2

The tissue taken out from medullary cavity of femur revealed confluent and diffuse replacement of the native hematopoietic cells and fat spaces of the marrow by histiocytes with abundant pale to eosinophilic cytoplasm. The nuclei are vesicular with dispersed chromatin and inconspicuous nucleoli. Occasional nuclear grooves, infoldings and lobulations are discerned. The morphology was consistent with the Langerhans Cell Histiocytosis. Thus, Langerhans Cell Histiocytosis or Class I histiocytosis with multifocal involvement without organ dysfunction was established and managed on its line in consultation with medical oncologist. Child was started on vinblastine, prednisolone and $6 \mathrm{MP}$ according to $\mathrm{LCH}$ risk group. Blood transfusion was given twice for decrease of haemoglobin due to cytotoxic drugs. The anemia, lymphadenopathy, hepatosplenomegaly and pain over the lower limbs responded to treat-meant. There was shrinking of the lytic lesions on radiography with the initiation of therapy. Child is on remission and is on regular follow up for any exacerbations.

\section{Discussion}

Langerhans cell histiocytoses are a group of seemingly diverse diseases all characterized by the abnormal accumulation of inflammatory histiocytes. The severity of manifestations and the duration of illness in histiocytoses vary from benign and self-limited to acute, disseminated and fatal. Jaffe and Lichtenstein first described eosinophilic granuloma in $1944^{8}$. Lichtenstein observed the similarities between eosinophilic granuloma, Hand-Schuller-Christian disease and Letterer-Siwe disease and proposed that they be considered as variants of a single process collectively known as "Histiocytosis " $\mathrm{x}$ " also known as Langerhans cell histiocytosis. Presence of proliferating histiocytes is the histologic hallmark of Langerhans cell histiocytosis, including eosinophilic granuloma. Morphologically these disorders are characterized by proliferation of mononuclear and sometimes-multinucleated cells with pale, ill-defined eosinophilic cytoplasm and lobulated nuclei with longitudinal grooves. Electron microscopy used for definitive diagnosis shows distinctive structures called Langerhans or Birbeck granules. These are rod like structures, with a striated core that may have a dilated end, giving them a tennis racket appearance. The Birbeck granules are organelles seen singly or in small clusters in the cytoplasm or as invagination of the plasma membrane and they are believed to be products of internalization of complexes derived from antigen at the cell membrane. Expression of CD1a by immunohistochemistry is also considered diagnostic of Langerhans cell histiocytosis $^{10,11}$.

Recently, histiocytosis has been classified into three classes $^{12}$. Class I and Class II are non-malignant whereas Class III is malignant. Our patient had manifestations of Class I or LCH in the form of multiple lytic lesions, lymph-adenopathy, hepatosplenomegaly and severe anemia without organ dysfunction. Children with organ dys-function have a higher mortality as compared to children with multiple organ involvement without dysfunction ${ }^{13}$. Bone involvement is very common in $\mathrm{LCH}$ but the reported incidence of isolated skeletal disease has not been uniform. In the large French series, 31\% presented with isolated uni or bifocal bone lesions and $19 \%$ had multifocal bone involvement ${ }^{14}$. The clinical course of $\mathrm{LCH}$ in patients with single system disease is generally benign with high chance of spontaneous remission and favorable outcome ${ }^{5,6}$. Bony lesions require simple curettage or even a liberal biopsy. Infiltration of affected site with steroids recently has also been reported to be an effective and safe modality ${ }^{15}$. Radiotherapy is reserved for disease that is inaccessible to intra-lesional injection of steroid or that is potentially compromising vital structures (e.g., optic nerve, spinal cord). In such case, a short course of radiation (450 cgy to $600 \mathrm{cgy}$ ) may prove effective. When multiple bones are affected, treatment generally consists of systemic agents as in our patient. When treatment of one site is clinically necessary, surgical removal, local injection of steroids or irradiation may be considered and treatment with potentially toxic systemic agents may be avoided. There is a significant difference in the overall survival and disease free survival. This is because recurrences, even if they are multiple do not lead to mortality. Even though, there is a suggestion that $\mathrm{LCH}$ is clonal disorder, its behavior is different from that of a classical malignancy.

There have been numerous efforts to define predictive factors that would allow separation of children expected to have a favorable course from those with fulminant disease. The best prognostic parameters currently available are age, extent of disease and presence of organ dysfunction (OD) as defined by Lahey in $1975^{16}$. It was generally agreed that disease confined to bone or lymph node had a good prognosis with minimal therapy and was associated with little morbidity. Those patients with multi-system disease fared less well, had a chronic course, high rate of morbidity and possible mortality. Prognosis of young children who had extensive disease involving many organs particularly those 
with dysfunction of vital organs was very bad with a high mortality irrespective of the treatment given. Although overall survival is good, recurrences are seen even in patients with single system disease at diagnosis. The role of chemotherapy in preventing these recurrences is questionable.

In conclusion, the case is highlighted which presented to us as unexplained severe anemia without any clue to the diagnosis. But later development of organomegaly, lymphadenopathy and especially X-ray features of multiple lytic bony lesions helped us to reach the diagnosis. Therefore, LCH has a broad clinical spectrum from; isolated cutaneous, pulmonary or bone localisation to acute widespread cutaneous and visceral disease. It may present in a wide variety of ways to a number of different specialists, and is often initially misdiagnosed or lately diagnosed.

\section{Acknowledgement}

Sincere thanks to Dr.Arjun Shrestha(Associate Professor, Dept. of Orthopedics) for kindly taking out the tissue from lesional site (Left femur), Dr. A Mitra (Associate Professor, Dept. of Pathology) for examination of tissue and confirming the LCH. The author would like to acknowledge the child and the parents for the immense help and co-operation for all the painstaking task and who kindly consented for publication. The authors are thankful to the Director and the hospital management for granting permission to publish the case report.

\section{References}

1. D'Angio GJ, Favora BE, Ladish S. Workshop on the childhood histiocytosis X: Concepts and controversies. Med Pediatr Oncol 1986; 14: 104-117.

2. Osband ME, Lipton JM, Lavin P, Levey R, Vawter G, Greenberger JS. Histiocytosis X. Demonstration of abnormal immunity, $\mathrm{T}$ cell histamine $\mathrm{H} 2$ receptor deficiency and successful treatment with thymic extract. N Engl J Med 1981; 304: 146-153.

3. Willman CL. Detection of clonal histiocytes in Langerhans cell histiocytosis: Biology and clinical significance. Br J Cancer 1994; 70(Sapp 23); S29-S33.

4. Broadbent V, Gadner H. Current therapy for Langerhans cell histiocytosis. Hematol Oncol Clin North Am 1998; 2: 337-338.
5. Rivera-Luna R, Martinez-Guerra G, Altami-ranoAlvarez E, Martinez-Avalos A, Cardenas-Cardoz R, Ayon-Cardenas A, et al. Langerhans cell histiocytosis: Clinical experience with 124 patients. Pediatr Dermatol 1988; 5: 145-150.

6. Raney Rb, D' Angio GJ. Experience at the Children Hospital of Philadelphia: 1970-1984. Med pediatr Oncol 1989; 17: 20-28.

7. Chu T D, Angio GJ, Favara B, et al. Histiocytosis syndromes in children. Lancet 1987;i:208

8. Jaffe HL, Lichtenstein L. Eosinophilic Granuloma of Bone. Arch Pathol, 1944; 37: 99

9. Smirniotopoulos JG, Olmsted WW. Primary and Secondary Neoplasms of the Skull. In: Putman CE, Ravin CE,

10. David R, Oria RA, Kumar R, et al. Radiologic features of eosinophilic granuloma of bone. AJR, 1989; 153: 1021.

11. Borrego O, Barness EG. Eosinophilic Granuloma (Langerhans Histiocytosis). Arch Pediatr Adolesc Med. 1998; 152: 91-92.

12. Mahmod H, Wang WC. The Histiocytosis. In: Rudolph's Pediatrics, 20th edn. Eds. Rudolph AM, Hoffman JIE, Rudolph CD. New Jersey, Prentice Hall, 1996: pp 13021306.

13. Kusumakumary P, Priyakumari T, Chellam VG, James FV, Nair MK. Langerhans Cell Histiocytosis in children less than 2 years of age. Indian Pediatr 1999; 36: 2935 .

14. The French Langerhans cell Histiocytosis Study Group. A multicentre retrospective survey of LCH: 348 cases observed between 1983 and 1993. Arch Dis Child 1996; 75: 17-24.

15. Wolfson SL, Botero F, Hurwitz S, Pearson HA. Pure cutaneous histiocytosis X. Cancer 1981; 48: 22362238.

16. Lahey ME. Histiocytosis X: An analysis of prognostic factors. J Pediatr 1975; 87: 184-189. 